\title{
Working process for installing snow melting plants with active working element
}

\author{
Anatoly A. Serebrennikov ${ }^{1, *}$ and Andrey A. Plohov ${ }^{1}$ \\ ${ }^{1}$ Tyumen Industrial University, 625027, Tyumen, Russian Federation
}

\begin{abstract}
Based on the operating experience analysis of snow melting plants and the proposed patent solutions for their improvement, a new design is developed. Operability of the new design, in which rotation of the working bodies is carried out under the influence of the supplied coolant's pressure, is confirmed. The study of the working process is based on an experimental method based on physical modeling. The influence of individual factors affecting the intensity and efficiency and enabling to determine the dimensionless similarity equations, is studied. Based on the results obtained, a pilot plant is designed, created, and tested under real operating conditions.
\end{abstract}

\section{Introduction}

A significant part of the territories of the Russian Federation is located in climatic zones with a long winter period. Currently, in case of heavy rainfall in the form of snow, in cities, especially large ones, some difficulties arise in its timely cleaning. The lack of proper technical means to ensure the implementation of the required measures leads to difficulty, and sometimes to a complete stop of traffic flows. Statistical data confirms that in the days of snowfall (up to $30-40 \%$ ) the number of road traffic accidents and domestic injuries increases significantly. These problems are characteristic not only of Russia, but also for a number of European countries (Finland, Sweden, Denmark, etc.), Canada, and individual US states [1, 2].

The range of technical equipment intended for cutting, grinding, collecting, loading and other operations related to clearing life-supporting areas from snow and ice cover [3, 4], gradually, but with a trend of increasing distribution, includes snow melting plants (SMP) of various performance.

The feasibility of using SMP is confirmed by a reduction in transportation costs. With an optimum arrangement of the plants, the distances from snow collection sites to disposal sites are significantly reduced. However, the main effect is associated not with minimizing transport costs, but with the environmental aspect.

Currently, various methods are used to counteract winter ice, including those based on the use of salt and multicomponent chemicals. Even with a complete rejection of them, it is known that the products of emissions accompanying the operation of vehicles, the activities of manufacturing enterprises and other types of anthropogenic influences accumulate in the

\footnotetext{
*Corresponding author: sereb_a_a@mail.ru
} 
snow $[5,6]$. Thus, as a result of the removal of snow masses collected from the city streets, the areas with extremely high concentration of pollutants are formed in places of its dumps [7].

The use of SMP minimizes these negative manifestations. After melting of the snow-ice mass, its discharge into the sewer systems is provided. Further, through the pipeline engineering communications, the formed liquid phase enters the city sewage treatment plant with the subsequent separation of harmful impurities.

\section{Status of the matter and research methodology}

In order to study the dynamics of the snow mass melting process, a number of experimental studies were carried out based on observations of the phase transition intensity [8].

The assessment of changes in temperature parameters based on the analysis of the accumulated bank of thermograms (Fig. 1) obtained using a thermal imager enabled us to develop a recommendation to ensure the possibility of phase transition intensification.

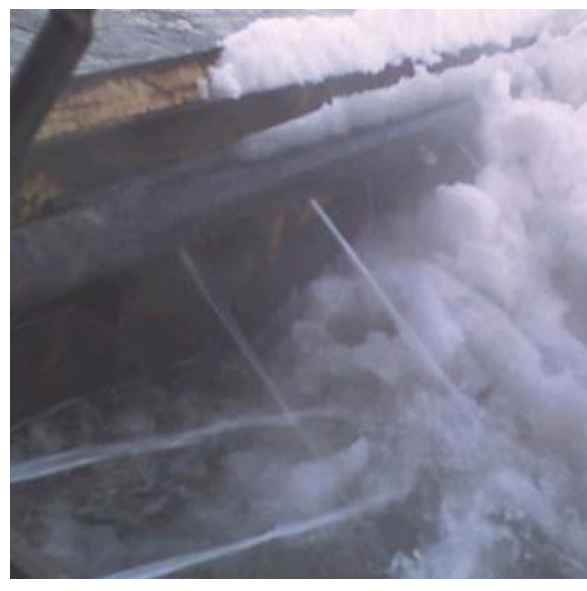

a)

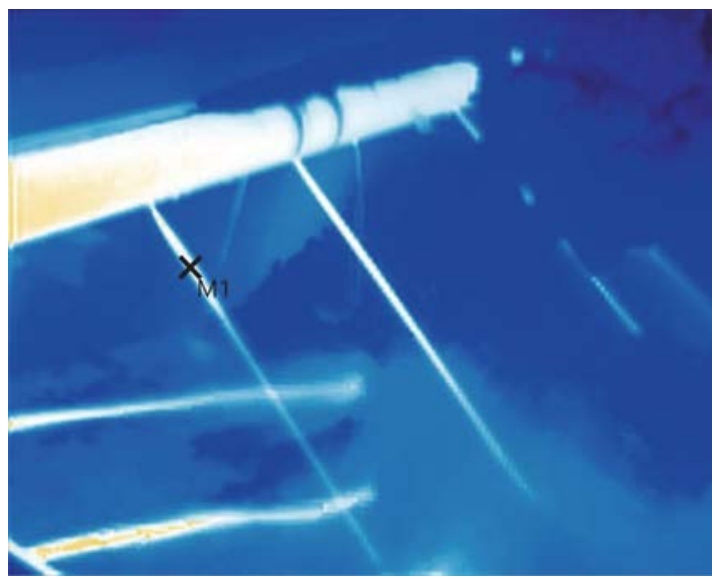

b)

Fig. 1. A typical frame of workflow recording: a - general view, b - infrared image (with a thermogram)

The essence of the proposal lies in the fact that it is necessary and possible to achieve an increase in the contact area of snow with the coolant due to the continuous destruction of the molten mass and the supply of coolant to its depth [9].

A distinctive feature proposed for the implementation of the technical solution [10] is a combination of two directions of workflow intensification in one device.

This result is achieved because the coolant is supplied into the melted snow mass by active working bodies, which are hollow shafts on which tubes with spray nozzles are installed.

In the proposed device, rotation of the active working bodies is carried out due to the reaction of the jets flowing from the tubes (application of the 'Segner wheel' principle). Thus, the pressure created in the circulation system is used, and there is no need for additional drive systems for moving the working bodies.

The performance check of the proposed technical solution and the assessment of the workflow running in it were carried out on physical models.

The main goal of the research was to determine the intensity and efficiency of the working process of the proposed SMP design. In this case, the time taken to ensure the transition of the snow mass into the liquid state is taken as intensity. Efficiency is 
understood as achievement of the named technological result at the expense of certain work. It is obvious that the work process of SMP will be more effective, if less work is spent on the phase transition.

The research is based on an experimental approach based on physical modeling.

To carry out the first stage of research, a physical model with a constant of geometric similarity $\left(\mathrm{C}_{\mathrm{V}}=15\right)$ in relation to the planned production version of SMP was created. The capacity of a loading hopper was $\sim 0.5$ cubic meters $(0.8 \mathrm{~m}$. x $0.8 \mathrm{~m}$. x $0.8 \mathrm{~m}$.).

The parametric calculation of the stress-strain state and the visualization of the physical model were carried out using 3D CAD (SolidWorks). The framework, sheet elements, pipes, and the layout of all structural elements were calculated (Fig. 2).

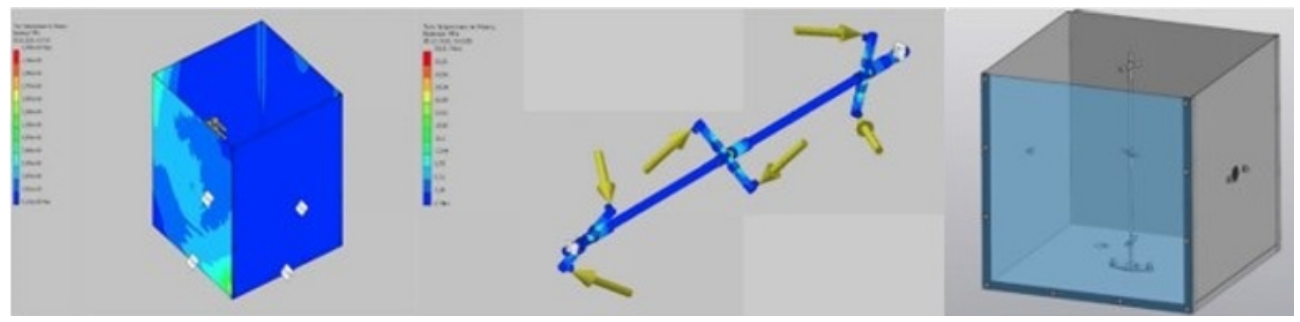

Fig. 2. Calculation and visualization elements using $3 \mathrm{D} C A D$

To be able to observe the dynamics of the snow mass melting process, the front wall of the hopper was made of organic glass. The pressure of the circulating water (coolant) created by the pump was recorded by a manometer and regulated by a valve installed on the bypass line of the pipeline system.

Before filling the tank with snow, the working pressure necessary to overcome the resistance arising in the rotary joint before the start of the working body rotation was determined. Its value was about $0.15 \mathrm{MPa}(1.5 \mathrm{~atm}$.).

In accordance with previous justifications [9], the average density of loaded snow was $600 \mathrm{~kg} / \mathrm{m}^{3}$ ( $\left.\pm 7 \%\right)$. The initial water temperature supplied to the circulation was kept constant $-30{ }^{\circ} \mathrm{C}( \pm 10 \%)$.

A series of experiments proved the efficiency of the proposed design. The working body began to rotate almost immediately after overcoming the initial moment of resistance in the rotation joint, cutting the snow massif into layers.

The physical model's design provided for the possibility of measuring the amount of snow remaining in the hopper, depending on the duration of work and, thereby, assessing the melting process intensity.

For this purpose, guides were made from welded corners on the inner side walls of the tank at the distance of every 10 centimeters. After stopping the flow of coolant, the transparent front wall was removed. A pre-prepared sheet of organic glass was inserted into the grooves of the guides and a slice was made - separation of the snow layer with its subsequent selection and weighing. Recording the amount of snow in each cut layer (from the periphery to the center of the massif) made it possible to evaluate the depth of melting. The ratio of the total remaining mass to the amount of initially loaded snow showed a phased intensity of the phase transition. In addition, a photofixation of the nature of the change in the shape and volume of the snow massif located in the influence zone of the working body's nozzles was made (Fig. 3).

A series of such experiments with a change in the stopping time from the start of the process (at intervals of 30 seconds) was carried out.

The calculated amount of molten snow was used as a parameter of indirect estimation of the energy spent on the phase transition. 
During SMP operation, the structural and mechanical properties of snow change as a result of the transition of the material from solid to liquid. The basis for obtaining the dimentionless equation describing the process became a dimensional analysis method. The parameters that affect the nature of the workflow are further identified, and the dimensional functional analysis using the $\pi$-theorem yields a general functional relationship between similarity criteria.

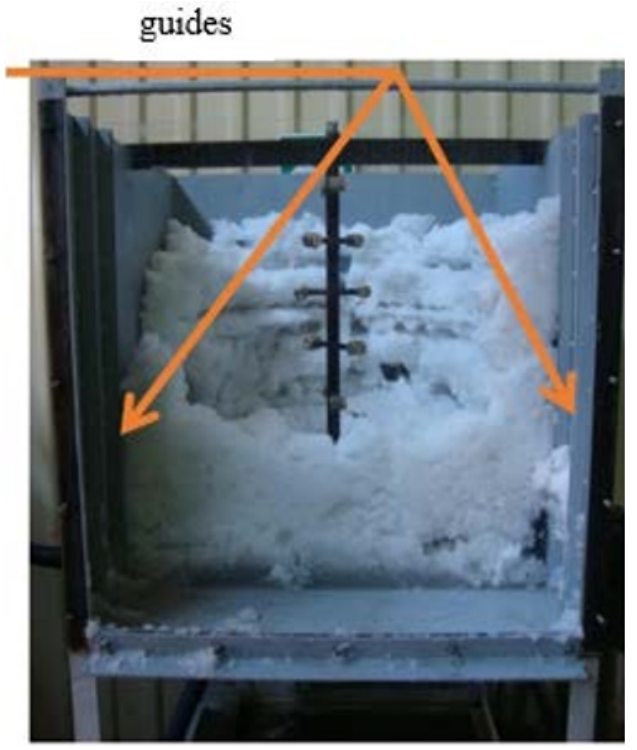

a)

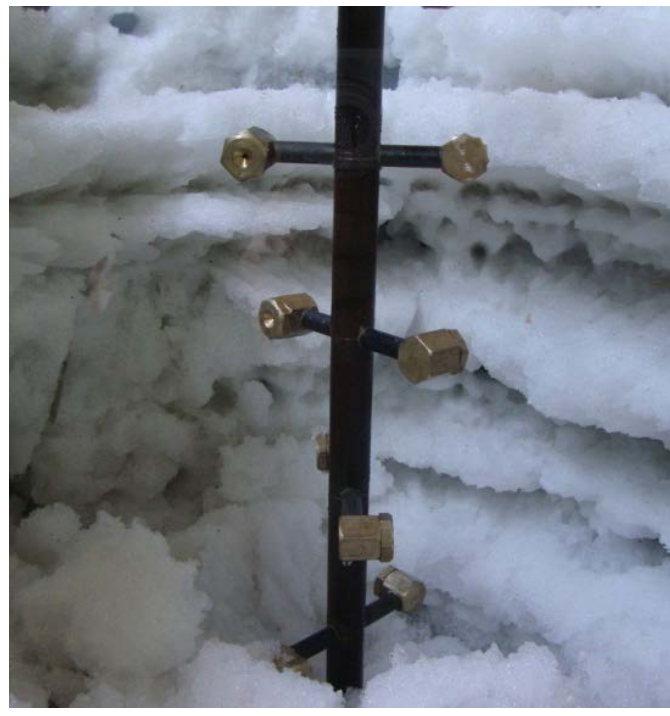

b)

Fig. 3. General types of measurement procedure: a - layered sections, b - recording the nature of impact on the array

To establish the nature of the criteria's influence, production of another physical model was required.

The second physical model was created with a constant of geometric similarity $\left(\mathrm{C}_{V}=3\right)$ in relation to the planned production version of SMP. The loading hopper's capacity was 2.5 cubic meters $(1.2 \mathrm{~m}$. $\times 2.0 \mathrm{~m} . \times 1.0 \mathrm{~m}$.). In accordance with the results of the experiments, the hopper was equipped with two active working bodies with 12 nozzles (each), providing a spray torch of 45 degrees.

The worst conditions for loading SMP are determined by the maximum average density of loaded snow $\left(600 \mathrm{~kg} / \mathrm{m}^{3}\right)$. If we take this indicator as the initial one and assume that the working process is ensured by a stable coolant temperature $\left(30^{\circ} \mathrm{C}\right)$, then for the structure under study the intensity and efficiency will depend on the created volumetric flow rate of the coolant flow.

In addition, an assessment was made of the change in the intensity of the working process of the proposed SMP design (with active working bodies) in comparison with traditional types of coolant supply through the upper irrigation circuit (Fig. 4). 


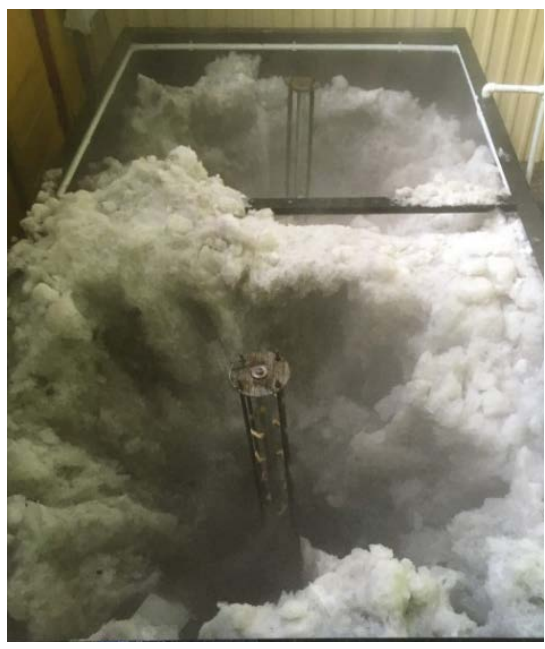

a)

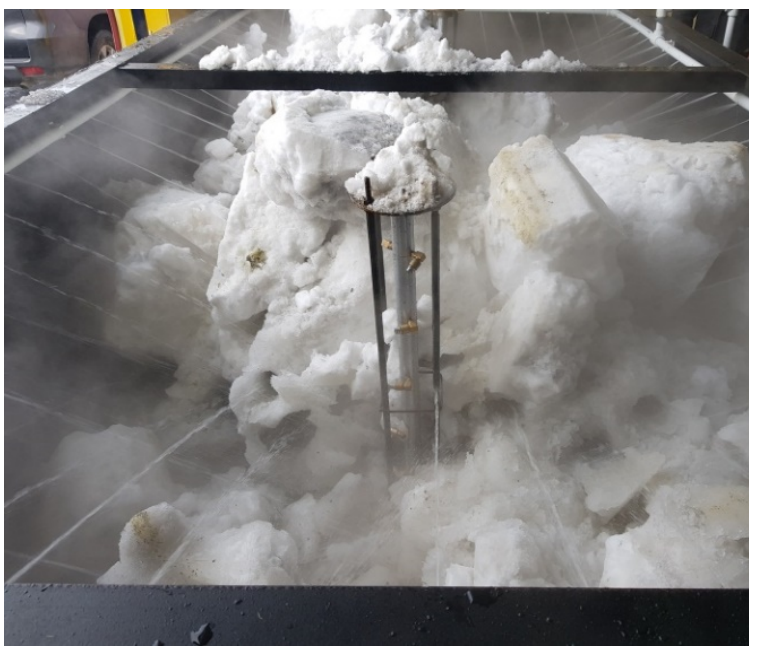

b)

Fig. 4. General types of the working process: a) coolant supply through the active working body, b) coolant supply through the upper irrigation circuit

\section{Conclusions, recommendations, results}

As a result of studying the dynamics of the SMP working process of a standard design with the coolant supply through the upper irrigation circuit, its low intensity due to the small contact area of the coolant with the snow is proved.

A new design of SMP with active working bodies providing the coolant supply from the snow massif depth is proposed.

Operability of the new design of SMP, where rotation of the working bodies is carried out under the influence of the pressure of the supplied coolant, is confirmed. It is proved that the magnitude of the force, as the resulting sum of the reaction of the jets escaping from the nozzles, is sufficient to overcome the arising resistances. Thus, special drive systems for working bodies are not required, which are proposed in a number of design solutions identified as a result of patent search and analysis.

Creation of SMP physical models and study of the working process on them using the basic principles of the theory of dimensions similarity and analysis made it possible to obtain the required quantitative characteristics.

In particular, the amount of snow turning into a liquid state at a constant value of the coolant temperature is determined.

It was further established that an increase in the spray pattern leads to a decrease in the intensity of exposure to the surface being treated in connection with the dissipation of energy in the coolant flow. On the other hand, a decrease in the spray angle will lead to the distancing from the working body of the point of flare intersection and to a decrease in the contact area of the coolant with the medium being treated. In case of jet outflow (there is no spray angle), the snow mass will simply be cut into layers, which is impractical.

It is recommended to establish a constant value of the coolant spray angle (45 degrees), regardless of the size of the designed SMP.

As a result of the comparison, it was found that the workflow intensity of the proposed SMP design (with active working bodies) is $25-30 \%$ higher relative to the intensity of typical structures (with an upper irrigation circuit). 
In the initial period of operation (after filling the hopper), a combined method of supplying coolant is advisable. However, after 15-20 minutes of operation, the upper irrigation circuit must be turned off, since its effect from a certain moment does not increase the intensity and, therefore, leads to unjustified energy costs.

Based on the reseach results and formulated recommendations, the enterprise manufactured an SMP with a hopper capacity of $~ 7.5$ cubic meters $(2.0 \mathrm{~m}$. x $3.7 \mathrm{~m}$. x 1.0 $\mathrm{m}$.). The dimensions of the pilot SMP were limited by the site's size reserved for its placement in a closed-cycle car wash. The room has all the necessary equipment for preparation and supply of a coolant, as well as water purification devices.

All the remnants of the ice mass collected from the territory of the enterprise, accumulated in bulk at the time of production and launch of SMP in operation, have been disposed of. The total operating time of the pilot SMP amounted to 42 hours. The productivity of the plant at a coolant temperature of $300^{\circ} \mathrm{C}$ was about 15 cubic meters per hour and was consistent with the design, confirming the validity of the conclusions and recommendations.

\section{References}

1. A. Laurihavicius, D. Cygas, Winter maintenance problems on the streets of Lithuanian cities: X PIARC International Winter Road Congress: Technical Report, Lulea, Sweden, Lulea, Vol. 3, 16-19 march (1998)

2. P. F. Keranen, Optimization of winter maintenance in the Minneapolis, St. Paul Metropolitan area using performance targets, XI International Winter Road Congress, Sapporo, Japan, Sapporo, 28-31 January (2002)

3. H. C. Deng, W. X. Ma, and B. D. Jing, Technology of removing snow and ice on roads and its developing trend, Construction Machinery and Equipment, v. 12, pp. 47-50 (2005)

4. J. Zhang, D. K. Das, and R. Peterson, Selection of effective and efficient snow removal and ice control technologies for cold-region bridges, Journal of Civil, Environmental, and Architectural Engineering, v. 3(1), pp. 1-14 (2009)

5. D. Sanzo and S. J. Hecnar, Effects of road de-icing salt ( $\mathrm{NaCl}$ ) on larval wood frogs (Rana sylvatica), Environmental Pollution, v. 140(2), pp. 247-256 (2006)

6. A. Nuorkivi, Allocation of Fuel Energy and Emissions to Heat and Power in CHP, EnergyAN Consulting, September, pp. 2-22 (2010)

7. H. Scharsching, Results of a field test of seven different commercial ice warning systems, Third International Symposium on Snow Removal and Ice Control Technology. Transportation Research Board, Minneapolis, Minnesota USA, 14-18 September (1992)

8. A. A. Serebrennikov, A. A. Plokhov, Impact of physical and mechanical characteristics of snow on the melting process intensity, IOP Conference Series: Earth and Environmental Science, v 272(2), URL: https://iopscience.iop.org/article/10.1088/17551315/272/2/022131

9. A. A. Serebrennikov, A. A. Plokhov, V. I. Panov, Increasing the intensity of the work process of snow melting installations, Ground transportation and technological complexes and means: materials of the international scientific and technical conference, pp. 283-288 (2017)

10. A. A. Serebrennikov, Sh. M. Merdanov, A. V. Sharukha, A. A. Plohov, Snow melting plant, patent holder: Federal State Budgetary Educational Institution of Higher Education "Tyumen Industrial University" (TIU), Pat. 189774, Russian Federation, IPC E01H 5/10, №. 2019100267, declared 01/09/2019, publ. 06/03/2019 